\title{
Framework for Interactive Data Mining Results Visualization on Mobile Devices
}

\author{
Muzammil Khan, Ali Shah, Israr Ahmad \\ Department of Computer Science \& Software Technology \\ University of Swat, KP Pakistan \\ muzammilkhan86@uswat.edu.pk, alishahedu@yahoo.com, \\ israrahmad009@gmail.com
}

\begin{abstract}
The rapidly improving technologies like data mining and mobile technology need careful investigation in order to emerge these technologies. In this paper we identified the challenges confront by mobile data mining, visualization challenges, and mobile device limitations. The paper introduced a comprehensive framework that explores the idea, how we can incorporate both of these promising technologies regarding the challenges facing mobile data mining and all possible application scenarios. The paper further specifies the possible ways to merge both of these technologies, with the available resources.
\end{abstract}

Keywords: Mobile data mining, Visualization, Framework, Mobile devices, Interactivity

\section{Introduction}

The internet and web services usage on mobile devices are continuously and rapidly increasing. Therefore the demand is to have efficient mobile interface that can effectively display information and efficiently utilize the small size mobile screen [1], low bandwidth and unreliable connection etc. Mobile devices, such as PDAs, Smartphones, Tablets etc evolve rapidly from the digital calendars and address book to hosts of more complex functionalities. Because of improving mobile devices resources, it supports and adapting all forms of application from computer systems. However mobility and scaled-down technologies lead to some limitations as compare to computer system [2]. Due to these limitations and deficiencies it is not straight forward to adopt or provide available facilities on mobile device as with desktop computers.

Data mining is one of the most emerging fields of computer science, and allows users to analyze large data or database to solve decision problems in business carpet. The use of improving mobile computing technologies supporting the data analysis techniques which leads to discover knowledge from every place in which people operate $[3,5,6]$.

The ubiquitous use of data mining techniques and rapidly development in mobile technology it is encouraging to introduce techniques to utilize these emerging technologies [4]. The use of data mining techniques using mobile devices is one of the challenging task, the shifting of expensive data mining task to mobile devices is confront by the limitations carry these devices. The researchers are busy to find out which techniques should be better to utilize mobile devices for data mining and facilitate the wide range of people using mobile devices to support them in their decision making and trend identification in huge data.

In incorporating these emerging technologies are; Data mining is expensive process, need high computing capabilities and Data are normally huge, which need high memory capacities. In contrast mobile devices have limited processing capabilities and memory capacities. 
Another important issue during data transformation between mobile device and data provider over low bandwidth wireless network is not feasible. Keeping in view the importance of data mining and the use of mobile devices, the demand is to effectively visualize results on mobile devices in such a way to provide information at a glance that is easily understood with less cognitive resources and distracts the user as less as possible from current task.

\section{Mobile Devices}

Mobile device is a small, easily portable handheld electronic device that provides some processing or computing capabilities. Also defined as pint-sized or runty computing device, also known as handheld device, handheld computer, or simply handheld.

Usually mobile devices come with mini keyboard of touch display. These pervasive (omnipresent) devices enable users to take advantage of computing power. Mobile devices are small in size, light in weight, having some processing capabilities, and are able to connect to the internet. These portable mobile devices vary a lot in terms of features and computing power i.e. cost, size, weight and battery life. Boot-up time also plays important role in the mobile device selection. Mobile device is also known as portable computing devices [7]. The word mobile device is used to describe a device that has the ability to connect to the internet and multi-functional devices. Mostly by mobile devices we mean Smartphones and Tablets [8].

Mobile devices are expected to rapidly evolve in the next few years in their processing speed, battery life and memory capacity, which will extend its utility in broader prospective.

\subsection{Mobile Device Features}

Significant features/characteristics associated with mobile devices are discussed bellow;

Computing Ability is one of the utmost important characteristic of mobile devices. Nowadays mobile device appear with computing capabilities. Mobile devices have information processing ability and follow the program instructions to perform the mathematical and logical operations.

Processing speed of Smartphones and Tablet is increasing day-by-day. Today we have 1.6 $\mathrm{GHz}$ Quad-core processors for Smartphone ${ }^{2}$, and $2.5 \mathrm{GHz}$ for Tablet available in market ${ }^{3}$. Primary memory is also an important component of a computing device. Smartphone carry upto $2 \mathrm{~GB}$ of RAM ${ }^{4}$, and Tablet carries upto $4 \mathrm{~GB}$ of primary memory ${ }^{5}$.

Storage Capacity: the capability of mobile devices to store data. Smartphone has storage capacity up to $32 \mathrm{~GB}^{6}$, and Tablet has storage capacity up to $64 \mathrm{~GB}$ or even more ${ }^{7}$.

Connectivity: Mobile devices have the ability to connect and communicate to other devices and to the internet. There are many options, Wi-Fi, Bluetooth, USB, microUSB, etc are different ways to connect and communicate.

Easily Portable: The small size of mobile devices enables one to carry it easily and usually use on the move.

Communication and Computing: Mostly mobile devices have the computing ability and also use to make calls. Mobile devices are capable to communicate with same nature devices, computer systems, cell phones and even access web sources via Internet using Bluetooth and Wi-Fi.

Support User Interaction: Mobile device support different user input and interaction for different interfaces, and also provide multiple functionalities. 
Rich Contents Display Ability: The most important interface between device users and machine is display screen. Today mobile device display screen support to present text, high quality graphics, animation, and video information.

\subsection{Mobile Devices Limitations}

Despite of rapid development in mobile technologies, mobile devices have potential differences as compared to conventional desktop computer systems. Many of these differences are in the form of limitations. The number of limitations or constraints discussed in [9] regarding mobile devices can be categorizing by the relevancy among these limitations. We can categorize into four different categories, that as Bandwidth or Network related constraints, Screen or Display related constraints, Hardware or Software constraints and Mobility or Location Related constraints.

\section{Network Constraints}

- Slow Connectivity

- Temporary Disconnection or Unreliable connection

- Low bandwidth

\section{Screen Constraints}

- Limited screen and small display

- Limited display and resolution

- Variable width and height ratio

\section{$H / W \& S / W$ Constraints}

- Limited processing capabilities

- Limited interaction techniques

- Limited input peripherals

- No full keyboard

- No mouse

- Limited memory and storage capabilities

- Device variability

- Limited graphical components and graphical libraries

- Complex and diverse data format

- Limited battery power or battery consumption

\section{Mobility Constraints}

- Auditory environment

- Visual environment

- Level of attention or concentration

\section{Challenges in Mobile Visualization}

The information visualization on mobile device face various challenges due to limited size screen and the absence of standard input devices, that make it more difficult to introduce new visualization techniques. To produce productive visualization techniques, we need to focus on the following; 
Information selection: As mobile devices have small screen so it is important to use it wisely and select those information which are utmost important to the users.

Information Presentation: To display useful information on small screen offers more challenges with mobile devices and become the most important problem to handle.

Interface Interactivity: To manage information on the available small screen need efficient and effective interactive mechanisms for the users.

Visualization features: To produce effective visualization, techniques need to have the visualization features like functionality, usability, effectiveness, efficiency and usefulness.

These are challenges on mobile devices as compare to personal computers. [9] and [10] comprehensively discussed visualization features, process, challenges in visualization, mobile visualization and other related concepts.

\section{Proposed Framework}

Mobile data mining is the revolution in the present era. The objective of mobile data mining is to facilitate the wide range of users with the complex data mining analysis techniques, to discover new knowledge any time any place using mobile computing technologies.

Mobile data mining faces many issues regarding mobile devices in mobile environment. Therefore, it is not simple to move high computing processes of data mining to the little processing capability mobile devices. Any solution for mobile data mining needs to keep in minds the mobile device limitations or constraints whether directly or indirectly involved in mobile data mining environment.

\subsection{Scenarios}

Data mining in the mobile environment may involve a number of application scenarios or situations. In these situations a mobile device can act as data provider, data analyzer, data client or a combination of any two or even a combination of all of them. The objects and their representation in all scenarios are briefly discussed below;

Data Provider: provide data for storage on some data repository or device, component, module that provide/generate data to data collection unit for mining.
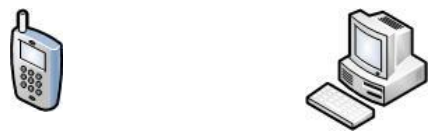

Figure 1. Data Providers: Cell phone and Computers

Data Analyzer: Device that acts as a mining machine and performs mining tasks. Device may be Server or Mobile device i.e. smart phone or tablet.
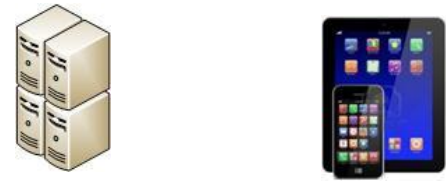

Figure 2. Data Analyzers: Application Servers and Advance high computing Mobile Devices 
Data Client: the device that request services from remote device or server. The services may be data, results of mining tasks or the way how to visualize the mining results.

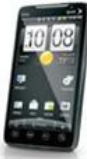

\section{Figure 3. Data Client: Mobile Devices}

Combination: By combination it means that the device may play many roles at a time, i.e., as data provider, analyzer, and as data consumer as well or any combination of it.

We can conceive six different mobile data mining scenarios, keeping in mind the role of mobile device.

\subsection{1. $1^{\text {st }}$ Scenario}

The data is stored locally on the server and mobile device act as a terminal for ubiquitous access. Remote server acts as data mining services provider. The mobile device requests sever for data mining task, the server performs mining on the data or distributed database and responds to the mobile device. In this scenario computers are considered to be data providers.
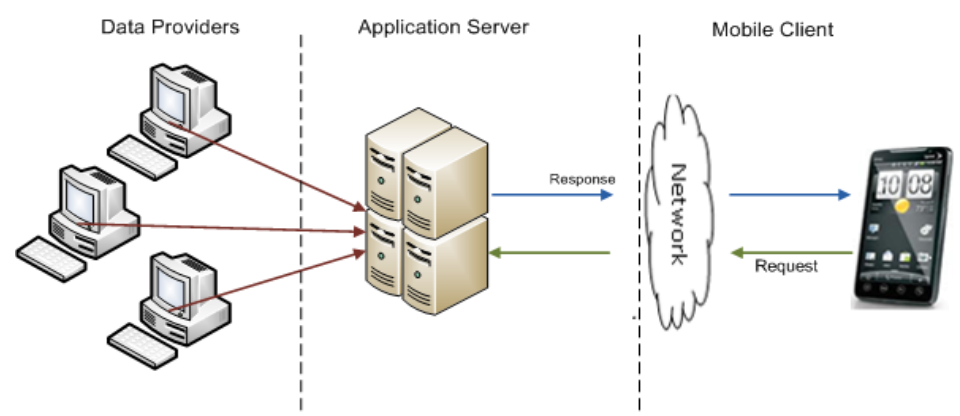

Figure 4. 1st Scenario; Mobile Devices as Client

Table 1. Mobile Devices as Client

\begin{tabular}{|l|l|l|}
\hline Data Providers & Server & Mobile Client \\
\hline Data providers & $\begin{array}{l}\text { Data Mining Services } \\
\text { Data Storage }\end{array}$ & Application interface (UI) \\
\hline
\end{tabular}

\subsection{2. $2^{\text {nd }}$ Scenario}

The data stored locally on the server, mobile device act as a terminal for ubiquitous access, remote server act as data storage. The mobile device requests sever for data, the server's streams data in response to the mobile device and the mobile device performs necessary data mining tasks. Due to constraints confronted mobile devices like computing power and data storage, it is not feasible for high computing. In this scenario computers are considered to be data providers, and best explain the following Figure 5 and Table 2. 


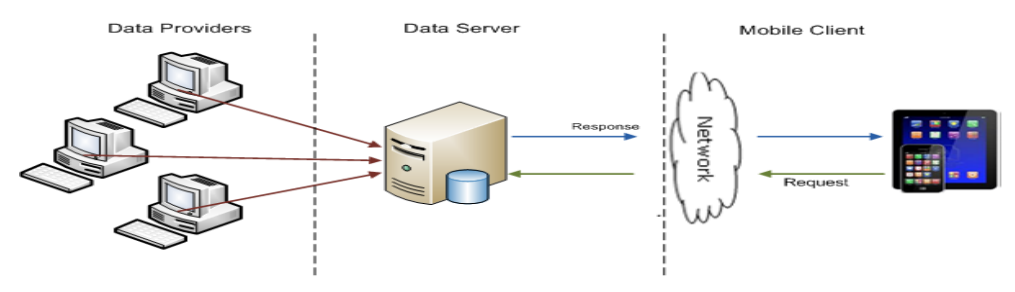

Figure 5. $2^{\text {nd }}$ Scenario; Mobile Devices as Data Analyzer

Table 2. Mobile Devices as Data Analyzer

\begin{tabular}{|l|l|l|}
\hline Data Providers & Server & Mobile Client \\
\hline Data providers & Data Storage & $\begin{array}{l}\text { Application interface (UI) } \\
\text { Data Mining Services }\end{array}$ \\
\hline
\end{tabular}

\subsection{3. $3^{\text {rd }}$ Scenario}

In this scenario, data is gathered via mobile device and stream it to the server for storage. Mobile device also acts as a terminal for ubiquitous access. The mobile device requests sever for data mining task, the server performs mining on the data or distributed database and responds to the mobile device. Mobile device acts as data provider and provides application interface.

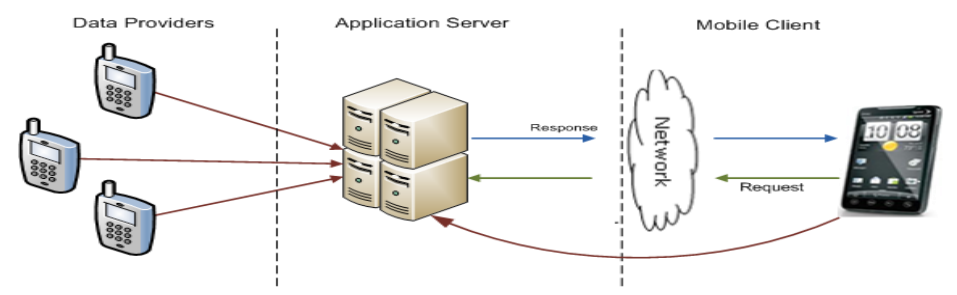

Figure 6. 3rd Scenario; Mobile Devices as Data Provider

Table 3. Mobile Devices as Data Provider

\begin{tabular}{|l|l|l|}
\hline Data Providers & Server & Mobile Client \\
\hline Data providers & Data Storage & Application interface (UI) \\
& Data Mining Services & Data providers \\
\hline
\end{tabular}

\subsection{4. $4^{\text {th }}$ Scenario}

Mobile device plays three important roles in this scenario. It provides application interface as data client, acts as data provider and also performs data mining tasks. Mobile device gathers data for server and requests data for mining operation about which the mobile device user is interested.

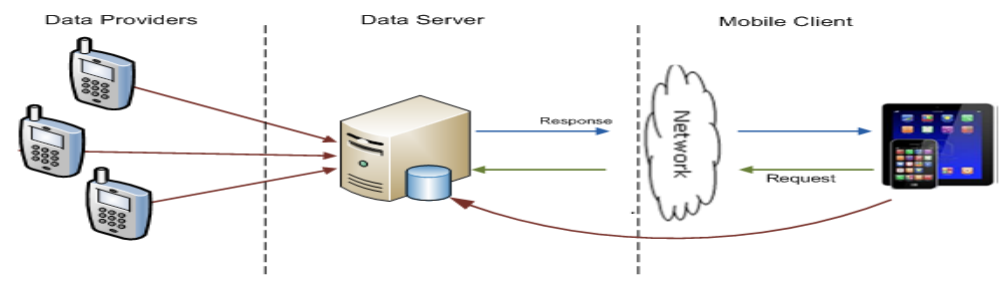

Figure 7. 4th Scenario; Mobile Devices as Data Provider and Analyzer 
Table 4. Mobile Devices as Data Provider and Analyzer

\begin{tabular}{|l|l|l|}
\hline Data Providers & Server & Mobile Client \\
\hline Data providers & Data Storage & $\begin{array}{l}\text { Application interface (UI) } \\
\text { Data providers } \\
\text { Data Mining Services }\end{array}$ \\
\hline
\end{tabular}

\subsection{5. $5^{\text {th }}$ Scenario}

In this scenario mobile device is considered to have sufficient amount of memory and high processing capabilities. Data mining processes are high computing processes, needing lots of computation power, and data sets are normally massive. Mobile device may store a part of data set rather than the whole data set while server keeps the complete data set.

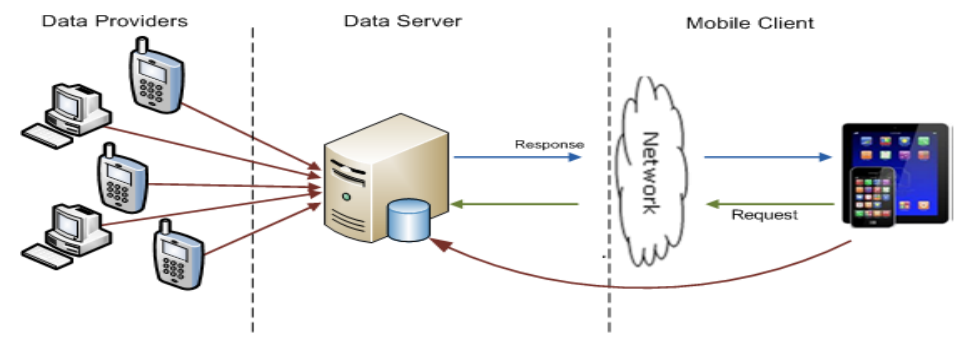

Figure 8. 5th Scenario; Mobile Devices as Data Provider, Analyzer and Storage

Table 5. Mobile Devices as Data Provider, Analyzer and Storage

\begin{tabular}{|l|l|l|}
\hline Data Providers & Server & Mobile Client \\
\hline Data providers & Data Storage & Application interface (UI) \\
& & Data providers \\
& & Data Mining Services \\
& & Data sets (few sets) \\
\hline
\end{tabular}

\subsection{6. $6^{\text {th }}$ Scenario}

This is very rich environment, which provides many options to the mobile device user and makes available almost all the possible alternatives. Mobile devices acts like resourceful devices, perform mining tasks, hold part of database and provide data as well. Server executes data mining task and data storage. Both client mobile device and server provides alternative services.

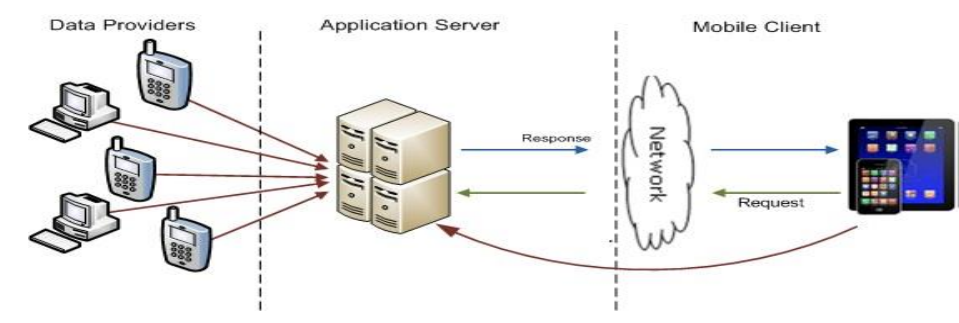

Figure 9. 6th Scenario; Mobile Devices and Server as Data Analyzer 
Table 6. Mobile Devices and Server as Data Analyzer

\begin{tabular}{|l|l|l|}
\hline Data Providers & Server & Mobile Client \\
\hline Data providers & Data Storage & Application interface (UI) \\
& Data Mining Services & Data Mining Services \\
& Data Storage & Data providers \\
& & Data sets (few sets) \\
\hline
\end{tabular}

The terms used in mobile data mining environment are;

Data Sets: Any named group of records is called a data set. The term data set refers to a file that contains one or more records ${ }^{8}$.

Data Mining Services: The actual data mining task is the automatic or semi-automatic analysis of large quantities of data to extract previously unknown interesting patterns. Network: Medium through which mobile, server, computer system interacts or communicates in mobile data mining environment.

Data Analyzer: Device that acts as a mining machine and performs mining tasks. Device may be Server or Mobile device i.e. smart phone or tablet.

Application Interface or Front End: The medium through which end user requests for data mining results and invoke data mining tasks on data stored on server. The source provides presentation services to data consumer'.

Data Provider: It is a device, component, module that provides data to data collection unit, for storage to some data repository.

In order to incorporate emerging technologies of mobile devices and data mining techniques, one needs a thorough examination of all the six scenarios and selects the best one for time being (in current situation and available resources). Keeping in mind the mobile device constraints, massive data sets, and high computing data mining techniques or processes, 1st and 3rd scenarios are best to employ.

In both the scenarios mobile device acts as a client. The client mobile device invokes and performs data mining task on available data on the server, requests for the significant results and visualize it in some useful form on the mobile device.

\section{Architecture Design}

This section describes the architecture of the proposed framework. The figure shows the main components and their interconnection involved in the mobile data mining environment scenarios.

\section{Layered Architecture Design}

The Figure 10 shows the layered architecture of the proposed framework. The figure shows the main components, their interconnection and the sequence of functions performed by the components involved in the mobile data mining environment scenarios.

\section{Data Collection Layer}

Data collection layer is the first layer of proposed architecture. The two main functionalities of data collection layer are; to collect data from a variety of sources and store that data with relevant data. The two components of the layer are; Data collection unit and Data repository. 


\section{Data Pre-Processing Layer}

The data is originated from heterogeneous multiple sources may contain some missing values, inconsistent and noisy data. To get high quality pure data, it requires filtration. Data pre-processing layer provide functionalities to produce high quality data.

\section{Data Mining Layer}

The data mining layer performs data mining tasks on the data provided by the previous layer. The data mining results are communicated to the next layer.

\section{Results Manipulation Layer}

The data manipulation layer has two components; setting and specification unit and results repository. The results are handled in the specification unit according to the nature of the results produced and specify the best way to represent it. The results are stored in the results repository with specifications.

\section{Network Layer}

Network layer provides the medium between upper layers and lower layers in the proposed architecture.

\section{Results Transformation Layer}

According to the end user request query the results from the results repository at results manipulation layer will transform into graphical presentation according to the specification. The result management unit store results if user wish, which can be used then after.

\section{Presentation Layer}

Presentation layer provides user interfaces. The expert can manage the datasets, preprocessing, data mining operations, specifications etc represented via black color links in the figure 10. The end user can interact with the system using end user interface, can put query and view results via interactive visualization techniques.

\section{Component Architecture Model}

The components of the architecture can be divided into two models i.e., information extraction model and information visualization model, discussed below;

\subsection{Information Extraction Model}

The information extraction model is server based model and the tasks in the model will be performed on the server. The functionality of the component in information extraction model will be under the control of expert, who knows the data, preprocessing techniques, data mining techniques and the way how to visualize the information provided by the mining results to general users. The components are shown in figure 11 and its tasks are discussed briefly below;

\section{Data Collection Unit (DCU)}

The DCU is invoked to collect data from the data providers. The DCU interface accepts data to a set of different data providers and also has the mechanisms for data set operations, 
i.e., to upload new data set, delete or upgrade the existent one. The DCU transfers the data to store in the data repository.

\section{Data Repository (DR)}

The DR provides the list of locally available data sets.

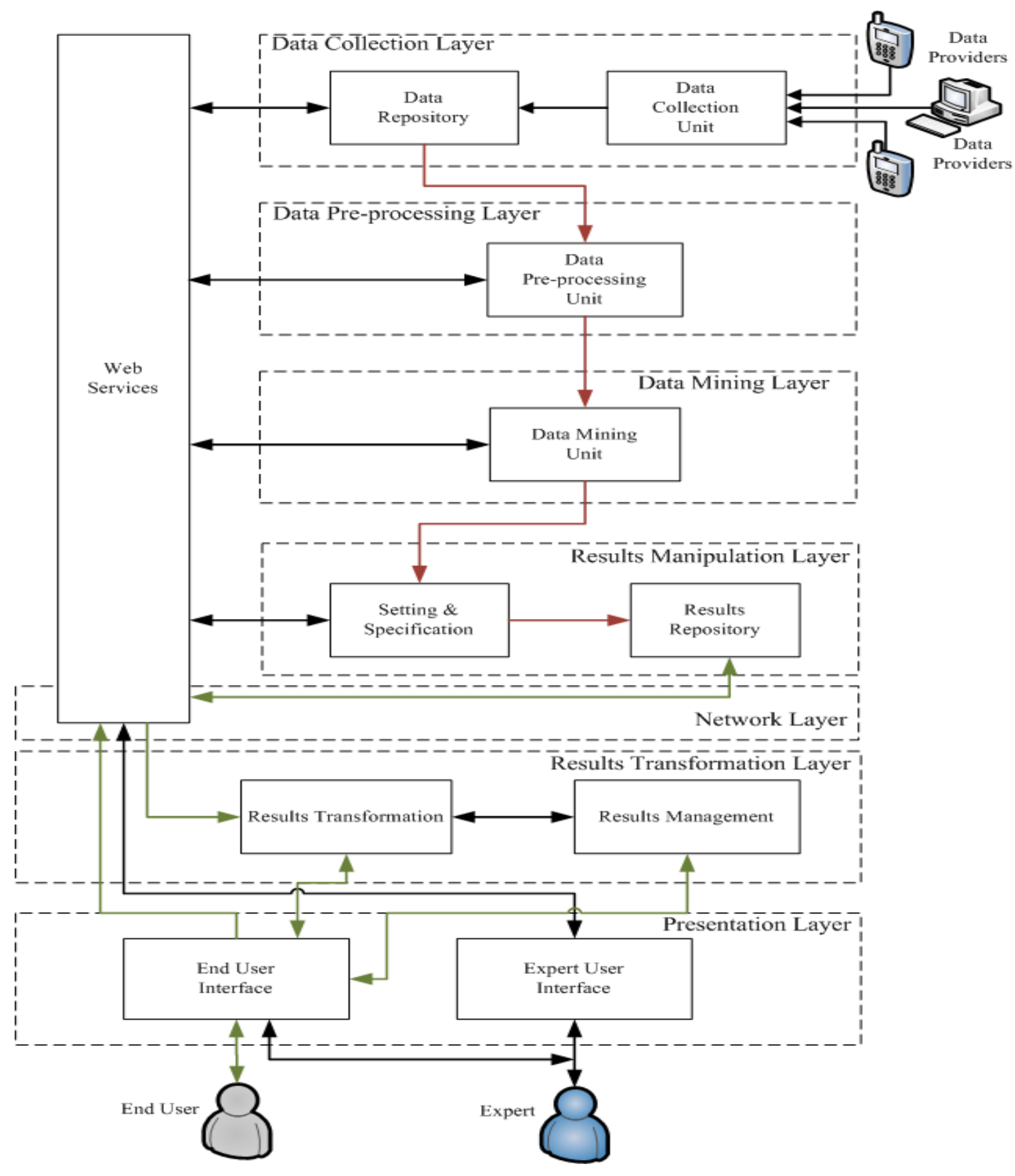

Figure 10. Layered Architecture of Mobile Data Mining Environment Scenarios 


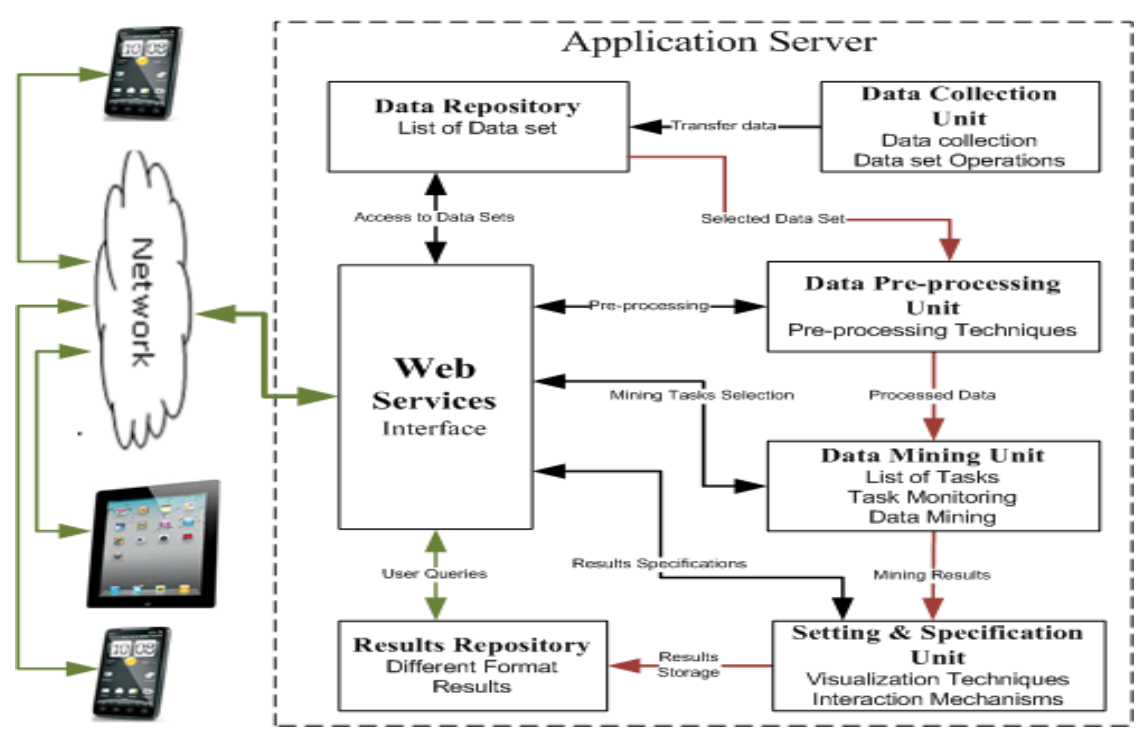

Figure 11. Information Extraction Model

\section{Data Pre-processing Unit (DPU)}

The DPU enables the expert to apply data pre-processing techniques in order to produce high quality data for data mining. Filter data to remove anomalies, inconsistency, redundancy etc., using data cleaning, normalization, reduction etc operations.

\section{Data Mining Unit (DMU)}

The DMU presents the list of mining tasks or algorithms, and enables the expert to invoke the operation and monitor i.e., to check status of tasks (ready, running, complete) and perform data analysis tasks.

\section{Settings and Specification Unit (SSU)}

The mining results need to be refined for the end users. The SSU enables the expert to set parameters for the result presentation i.e., visualization techniques for displaying results, interaction mechanism specification etc.

\section{Results Repository (RR)}

The RR keeps the results in a format that is readable to the web services. The results may be stored in database, $x m l$, textual file or any other readable format with their visualization specifications.

\section{Web Services (WB)}

Web services provide the interface between users (expert and end user) and functional components.

\subsection{Information Visualization Model}

The visual information model is mobile device client based model and the main task of the components involved in the model is to provide interactive usable data mining results 
visualization techniques on mobile devices i.e., smartphones and tablets. The Figure 12 shows information visualization model components and its tasks are discussed briefly below;

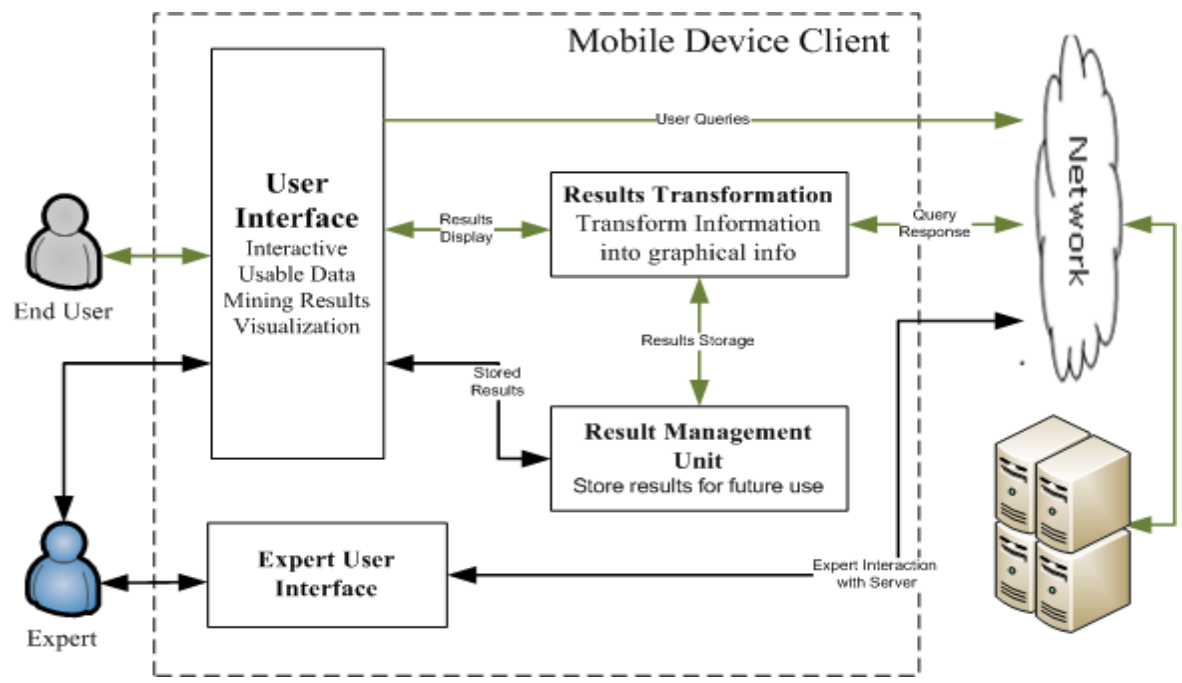

Figure 12. Information Visualization Model

\section{Results Transformation (RT)}

The RT unit transforms the information into some graphical objects by using some scripts, according to the specification made in the settings and specification unit (SSU). The end users can change visualization and interaction mechanism as specified.

\section{User Interface (UI)}

The UI provides facility through which user can interact, responsible to display information visually that is transformed by results transformation unit and interaction to the visualizations.

\section{Result Management Unit (RMU)}

The RMU is responsible to store results in image form which can be used in future.

\section{Expert User Interface (EUI)}

The EUI provides the facility to manage the components of information extraction model, and facilitates the mobile device end user with the benefits of data mining technologies to present the significant information by using interactive usable visualization techniques.

\section{Conclusion \& Future Work}

The paper focuses on comprehensive discussion on our proposed framework for the incorporation of data mining in the mobile devices environment. A section illustrates the potential scenarios where both data mining and mobile device technologies can be assimilate together. The paper discusses in detail the architecture of the proposed framework and layered architecture. The components of the architecture is divided into two component models i.e., information extraction model and information visualization model, which illustrated in their respective tasks and interconnection among different units. 
Considering all the factors described, the objectives for further research work is to develop a functional prototype in order to validate the proposed framework which facilitate mobile device users with interactive, useful and informative information in easy and effective way, which will deduce using expensive data mining operations, briefly discuss below;

\section{Dynamic Transformation of Layout}

Prototype should provide the facility to dynamically transform the visual layout according to mobile device.

\section{Scalable Layout}

The scalability of visualization techniques make it more feasible for small size screen, the zooming (zoom in or zoom out) should not distort the graphics and provide sharp and clear visual graphic display on mobile devices.

\section{Visualization Techniques}

The selection of visualization techniques depends on the nature of the data to visualize.

\section{Interactive Mechanisms}

The focus is on three interactive mechanisms.

Drill Down Approach: Access lower level details of the data from a higher level or drill down is to move from abstract or aggregated information to detailed information.

Legend Navigation Approach: The legend navigation approach is best suited for data discrimination i.e. to compare comparative target classes.

Drill down + Legend Navigation Approach: To get comprehensive details about target classes, means for both data characterization and data discrimination in one graphical representation both interactive techniques are used.

\section{Consideration of Visualization Features}

The prototype should support all the visualization features like functionality, usability, effectiveness, efficiency and usefulness.

\section{Multiple Brand Smart phones and Tablet Support}

The functional prototype should support different brands of smartphones and tablets regardless their underlying architecture (hardware and software). The data mining results can be presented as it is available, to a wide range of mobile device users, since web browser is being used for interactive visualization techniques presentation.

\section{References}

[1] H. Y. Yoo and S. H. Cheon, "Visualization by information type on mobile device," Proceedings of the 2006 Asia-Pacific Symposium on Information Visualization, vol. 60, (2006), pp. 143-146

[2] R. Sousa, V. Nisi and I. Oakley, "Glaze: A Visualization Framework for Mobile Devices," Human-Computer Interaction INTERACT, Springer (2009).

[3] U. Fayyad, P. Shapiro and S. Uthurusamy, "Advances in Knowledge Discovery and Data Mining," MIT (1996).

[4] A. K. Dubey and S. K. Shandilya, "Exploiting Need of Data Mining Services in Mobile Computing Environments".

[5] IEEE, International Conference on Computational Intelligence and Communication Networks, (2010) 
[6] U. Fayyad, G. Piatetsky-shapiro and P. Smyth, "From Data Mining to Knowledge Discovery in Databases," AI Magazine, vol. 17, (1996), pp. 37-54.

[7] J. Han, "Data Mining: Concepts and Techniques," Morgan Kaufmann Publishers Inc. San Francisco, CA, USA, (2005).

[8] C. Cebe, "Portable computing device", US Patent D612,376, (2010).

[9] R. Osterman, "Key issues to consider in mobile device management", (2011).

[10] M. Khan, "Interactive Data Mining Results Visualization on Mobile Devices", ISBN 978-3-659-46354-9, LAP Publisher USA, (2013).

[11] M. Khan and S. S. Khan, "Data and information visualization methods and interactive mechanisms: A survey", International Journal of Computer Applications, vol. 34, no. 1, (2011), pp. 1-14.

\section{Footnotes}

1. WebSter Dictionary: www.webster-dictionary.org/definition /aesthetic

2. Date: 10th Nov 2013, http://www.phonegg.com/Top/ Fastest-Processor-Cell-Phones.html

3. Date: 10th Nov 2013, http://tablets.findthebest.com/app -question/302/What-is-the-fastest-tablet-computer

4. Date: 10th Nov 2013, http://www.phonegg.com/Top/ Ram-Amount-Cell-Phones.html

5. Date: 10th Nov 2013, http://tablets.findthebest.com/l/ 47/Asus-EEE-Slate-EP121

6. Date: 10th Nov 2013, http://www.phonegg.com/Top/ Internal-Memory-Cell-Phones.html

7. Date: 10th Nov 2013, http://tablets.findthebest.com/1/ 19/HP-Slate-500

8. IBM http://publib.boulder.ibm.com/infocenter/zos/basics/index.jsp?zconc datasetintro.htm

\section{Authors}

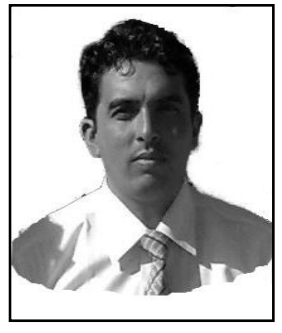

Muzammil Khan, Lecturer, Department of Computer Science \& Software Technology, University of Swat. I Received BS Computer Science Degree (Gold Medalist) Session 2005-09 from University of Malakand, MPhil Computer Science Degree Session 2010-12 from Quaid-i-Azam University Islamabad and Currently doing PhD Computer Science from Preston University Islamabad, Pakistan. Research interests are Human Computer Interaction, Usability, Data Mining on Mobile devices, Visualization, Mobile Visualization and Wireless Ad hoc Networks.

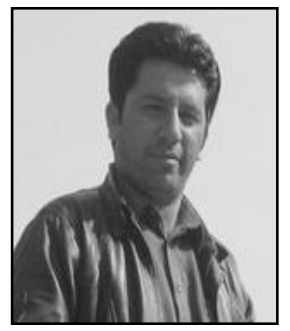

Ali Shah, received BS (Computer Science) degree from University of Malakand, Pakistan in session 2005-09, and currently doing MS in Computer Science session 2012-14 from Abasin University, KPK, Pakistan. His research interests in Human Computer Interaction and Visualization.

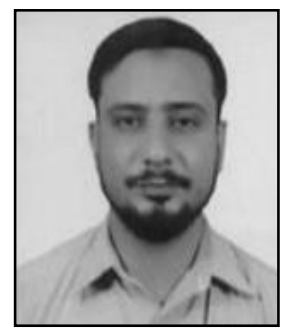

Israr Ahmad, received BS (Computer Science) degree from University of Malakand, Pakistan in session 2005-09, and currently doing MS in Computer Science session 2013-15 from Preston University, Islamabad, Pakistan. His research interests in Algorithms, Human Computer Interaction and Visualization. 